\title{
Large Functional Variability in Cockroach Photoreceptors: Optimization to Low Light Levels
}

\author{
Kyösti Heimonen, Iikka Salmela, Panu Kontiokari, and Matti Weckström \\ Department of Physical Sciences, Division of Biophysics, and Biocenter Oulu, University of Oulu, FIN-90014 Oulun yliopisto, Finland
}

The compound eyes of insects contain photoreceptors in small eyelets, ommatidia. The photoreceptors generally vary very little from ommatidium to ommatidium. However, in the large compound eyes of the cockroach (Periplaneta americana), previous studies have shown large differences in the optical structure between the ommatidia. The anatomy suggests pooling of $6-20$ photoreceptor signals into one second-order cell in the first synapse. Here, we show and characterize an unexpectedly large and seemingly random functional variability in the cockroach photoreceptors in terms of sensitivity, adaptation speed, angular sensitivity, and signal-to-noise ratio. We also investigate the implications of action potentials, triggered by the light-induced membrane depolarization in the photoreceptor axons. The combination of the functional features reported here is unique among the compound eyes. Recordings from the proximal parts of the thin and long photoreceptor axons or small and distant second-order neurons are not practical with the present methods. To alleviate this lack of data, we used computer simulations mimicking the functional variability, spike coding, and pooling of 12 photoreceptor signals, on the basis of our recordings from the photoreceptor somata and distal axons. The predicted responses of a simulated second-order cell follow surprisingly reliably the simulated light stimuli when compared with a simulation of functionally identical photoreceptors. We hypothesize that cockroach photoreceptors use action potential coding and a kind of population coding scheme for making sense of the inherently unreliable light signals at low luminance and for optimization of vision in its mainly dim living conditions.

Key words: vision; compound eye; graded responses; action potentials; population coding; modeling

\section{Introduction}

Sensory systems may be optimized for signaling under prevailing environmental conditions (Laughlin 1990; Weckström and Laughlin, 1995). The optimization can lead both to a large degree of accuracy and precision of responses to stimulation and consistency of responses in any group of neurons adapted to the same task. This is especially true for visual systems. In insect compound eyes, photoreceptors of any functional type have small variability (Laughlin and Hardie, 1978; Hardie, 1979; Eguchi and Horikoshi, 1984; Juusola et al., 1994), although no investigations on this aspect are available. The compound eyes may be classified into apposition eyes, where the small eyelets, the ommatidia, are optically isolated, and into superposition eyes, where this isolation is more or less deficient (Exner, 1891). The apposition eyes are considered to be ill suited for vision at low luminance. Most nocturnal insects have superposition eyes (Laughlin, 1990; Warrant et al., 2004). However, the apposition eyes of cockroaches apparently can cope with low light intensity conditions, and they

Received Aug. 30, 2006; revised Nov. 16, 2006; accepted Nov. 19, 2006.

This work was supported by grants from The Academy of Finland (M.W.) and Finnish Graduate School of Neuroscience (I.S.). We thank Eric Warrant for the possibility to do the receptive field measurements in his laboratory in Lund, Mikko Juusola for the use of his recording software (Biosyst), Eero Kouvalainen and Esa Luoma for their technical expertise, and several colleagues, especially Doekele Stavenga, Pasi Tavi, Eric Warrant, and Mikko Vähäsöyrinki, for their valuable comments on this manuscript.

Correspondence should be addressed to Matti Weckström, Department of Physical Sciences, Division of Biophysics and Biocenter Oulu, University of Oulu, P.0. Box 3000, FIN-90014 Oulun yliopisto, Finland. E-mail: matti.weckstrom@oulu.fi.

DOI:10.1523/JNEUROSCI.3767-06.2006

Copyright $\odot 2006$ Society for Neuroscience 0270-6474/06/2613454-09\$15.00/0 show morphological and functional adaptations to dim environments (Butler, 1973a,b; Butler and Horridge, 1973a,b; Smola 1976; Mote, 1990). Compound eyes have usually regular ommatidial arrays and a consistent function of photoreceptors (Stavenga, 1979; Laughlin, 1981; Shaw, 1990). In contrast, a fair amount of structural and functional variation has been found in cockroach photoreceptors. The morphology, optics, and photoreceptors of cockroach ommatidia are irregular (Butler, 1971, 1973a,b; Füller et al., 1989), leading to degradation of the sampled image (French et al., 1977). The anatomy and length of photoreceptor axons varies a lot (Ribi, 1977; Ernst and Füller, 1987), although they are all thin and long $(300-1500 \mu \mathrm{m})$ in comparison to most insects. The sensitivity and the width of their receptive fields also vary substantially between photoreceptors within the same adaptational state (Butler, 1973b). Again, in contrast to the other insects, cockroach photoreceptor axons produce spikes on their graded light responses (Weckström et al., 1993). Through modeling, it was estimated that only a small fraction of the amplitude of graded responses reaches the distant receptor terminals and that action potentials seem to be necessary in the axons.

Anatomical data suggest a large amount of pooling in the first visual synapse (Ribi, 1977; Ernst and Füller, 1987; Füller et al., 1989). The functional variability of cockroach photoreceptors, especially within the same adaptational state, has not been properly characterized before, and this characterization forms a major part of this paper. Here, we report that in cockroach photoreceptors a large accuracy, precision, and cell-to-cell consistency of the 
light responses does not seem to be the functional strategy. Instead, there are large variations in the properties of cockroach photoreceptors, unexpected when compared with other species. This raises the question, whether the variability of the light responses could be used in the optimization of vision at low luminance. As a first trial to answer this, we present a hypothesis in the form of a computer simulation, explaining benefits of observed variability and giving insight into optimization principles.

\section{Materials and Methods}

Animals and preparation. We studied the green sensitive photoreceptors of the adult male cockroach (Periplaneta americana) compound eye. A few females were also tested to ensure that the responses and their variability did not differ significantly from males. Cockroaches were maintained in a $12 \mathrm{~h} \mathrm{light} /$ dark cycle either at room temperature $\left(20-22^{\circ} \mathrm{C}\right)$ or at $25-27^{\circ} \mathrm{C}$. Recordings were done during different phases of the circadian rhythm and always at the room temperature. For capturing, the cockroaches were anesthetized with $\mathrm{CO}_{2}$. When the antennas and the legs started moving again, the cockroach was dissected behind the front legs. The front legs and the antennas were removed. To stop the interior of the head capsule from moving, we made a vertical incision between the eyes and a horizontal incision below the eyes. All of the cut surfaces and wounds were sealed with wax, and the remaining front part of the animal was fixed on the edge of a small glass plate with wax. Two small incisions were made on the dorsal corneal surface of the left compound eye so that a small sector of the cornea could be removed. To prevent dehydration, the formed opening was quickly covered with an emulsion of white Vaseline and water. The glass plate with the cockroach preparation was fixed on a cardan arm system so that the left eye was placed in the center of an imaginary sphere along whose surface the stimulation light could be moved. The grease-covered opening was positioned so that the recording electrode could either be directed to the photoreceptor somata in the retina or the axons below them. The reference electrode (a chlorided silver wire) was placed through a small incision in the cuticula or the cornea into the thorax or the other eye. The recording electrode was inserted through the opening into the eye tissue with a piezoelectric micromanipulator (PM 10; World Precision Instruments, Sarasota, FL). The experimenter controlled the direction and the location of the recording electrode by looking through a stereomicroscope (Leica, Wetzlar, Germany). Almost all of the recordings were done from the area of the black retina (i.e., the somata of the photoreceptors). Only some of the impulse responses were recorded from the photoreceptor axons (Weckström et al., 1993). After the preparation and electrode positioning the photoreceptors were left to dark-adapt for a period of $\sim 20-30 \mathrm{~min}$. Long periods of light stimulation were always followed by dark adaptation of at least several minutes.

Recording equipment and light stimuli. The voltage responses of the photoreceptors to light stimulation were recorded intracellularly with borosilicate glass capillary microelectrodes (resistances, 50-150 M $\Omega$ ) pulled with a Flaming/Brown puller (P-87; Sutter Instrument, Novato, $\mathrm{CA}$ ) and filled with a $2 \mathrm{M} \mathrm{KCl}$ solution, whose $\mathrm{pH}$ was adjusted to 6.84 with a K-phosphate buffer. The electrode capacitances were compensated and the recorded signals amplified with an intracellular amplifier (SEC-1L or SEC-05L; NPI, Tamm, Germany) equipped with a lowvoltage probe. Light stimuli were either pulses of given intensities and lengths or contrast noise sequences with desired contrast modulations at different mean adapting light levels. The stimulus voltage waveforms were originally produced, all of the measurements controlled, and the signals analyzed with a Matlab (Mathworks, Natick, MA)-based custommade software (Biosyst; courtesy of M. Juusola, University of Sheffield, UK) running on a computer equipped with a data acquisition card (DT2821; Data Translation, Marlboro, MA; or PCI-MIO-16E-4; National Instruments, Austin, TX). The light stimuli were produced either with a green high-intensity light-emitting diode (LED) (peak wavelength, 525 or $555 \mathrm{~nm}$ ) or a stroboscope (Cathodeon, Cambridge, UK), whose flash was guided to the cardan arm by a quartz cable. The stroboscope was used to produce $10 \mu$ s white light flashes for eliciting impulse responses. The LED was used when either longer light pulses or contrast

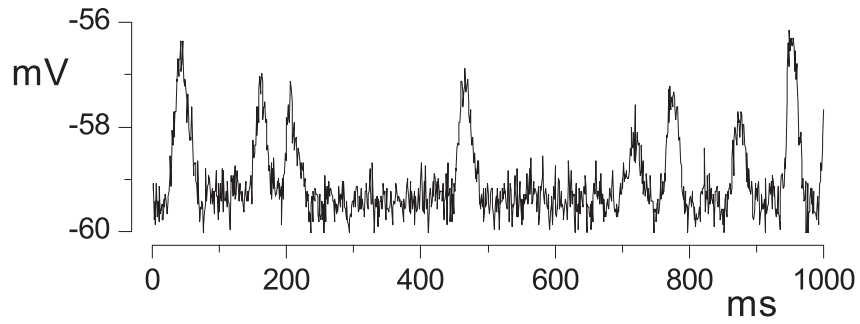

Figure 1. Calibration of stimulus light intensity. This example shows an intracellular recording of a cockroach photoreceptor, with eight single photon absorptions (i.e., quantum bumps per second).

noise was desired. The computer-generated stimulus waveforms for the LED were fed to a custom-made voltage-to-current driver, which controlled the current and thus the intensity modulation of the LED. The driver was equipped with a voltage output monitoring the LED current. If necessary, additional light intensity control was achieved by placing gray filters (Kodak Wratten; Kodak, Rochester, NY) between the stimulating light and the eye. The monitored stimuli and the amplified response signals were low-pass filtered with a matched channel filter (Kemo, Beckenham, UK).

Quality of recordings. The requirements of a photoreceptor soma to be accepted for the recordings were as follows: a sufficiently low resting potential (about -55 to $-65 \mathrm{mV}$ ), a high input resistance $(60-100 \mathrm{M} \Omega$ at resting potential), and clearly visible quantum bumps (amplitude, several mV) (compare Fig. 1), at a low stimulus light level. In the axon recordings, only the resting potential criterion was applied. When a cell recording fulfilled these requirements, the cardan arm was moved so that the maximal amplitude for the light responses was found. All of the recordings were done along this optical axis.

Recordings of photoreceptor sensitivity and adaptation speed. The stimuli used were either $300 \mathrm{~ms}$ or $10 \mathrm{~s}$ light pulses of desired intensities with long enough interstimulus intervals (ISIs) for proper dark adaptation (usually several seconds and with longer stimuli even minutes). The cell recordings were filtered at $700 \mathrm{~Hz}$ and sampled with $2 \mathrm{kHz}$.

Recordings of receptive field. These recordings were done with a different setup [courtesy of E. Warrant (University of Lund, Lund, Sweden)], described in detail previously (Warrant et al., 2004). In short, the setup was similar to ours, but the light source was a xenon arc lamp equipped with a quartz light guide and the cardan arm system was better suited for receptive field measurements. The operating curves [amplitude of voltage responses vs stimulus intensity (i.e., functional sensitivity)] and the horizontal receptive fields (i.e., angular sensitivity) of the dark-adapted photoreceptors were measured with impulse responses sampled at 2.5 $\mathrm{kHz}$ and at $1^{\circ}$ intervals. The ISI was always long enough to maintain the dark adaptation. The acceptance angles $(\Delta \rho$; the width of the receptive field at $50 \%$ sensitivity level) were determined from these sensitivity functions with linear interpolations between the recorded data points.

Statistical tests. To test the normality of the variability distributions, we used the Kolmogorov-Smirnov and the D'Agostino-Pearson test in MedCalc (MedCalc Software, Mariakerke, Belgium) and the Lilliefors test in Matlab.

Determination of signal-to-noise ratio. Photoreceptor signal-to-noise ratio (SNR) in the frequency domain was recorded and calculated as described in detail by Kouvalainen et al. (1994) (see also Juusola et al., 1994). Each cell, in which SNR was recorded, was first individually calibrated in terms of absolute sensitivity by counting the quantum bumps produced at a very low constant illumination (Fig. 1). The mean brightness of all of the adapting light levels used in the SNR recordings was calculated according to this calibration and the optical density of the gray filters $(0-5.0$ in steps of 0.5$)$ used in each case. Values of absorbed photons per second $(\mathrm{ph} / \mathrm{s})$ obtained this way are so-called effective values (Juusola et al., 1994, 1995). They are not absolute values (except at the calibration light level itself), because the quantum efficiency of the photoreceptors changes with the state of light adaptation (Smola, 1976; Wu and Pak, 1978; Laughlin 1981). However, the obtained stimulus intensity scaling is related both to the brightness of the stimulating light and to the 
sensitivity of the photoreceptor stimulated. Before starting a SNR recording session at a certain mean adapting light intensity, the cell was light-adapted for $2 \mathrm{~min}$, to allow the photoreceptor to reach a steady-state depolarization. When recording, a pseudorandom contrast noise sequence was repeated 20-30 times on top of each mean adapting light level. Each sequence record was $16 \mathrm{~s}$ long and had a contrast of 0.16 or 0.32 . Both the stimulus and the response were filtered at $500 \mathrm{~Hz}$ and sampled with $1 \mathrm{kHz}$. The time domain average of the responses gives an estimate of the signal, and any of the raw responses minus the signal is considered noise. The spectra of the signal and the noise were calculated from 2048 point segments with $50 \%$ overlap using a Blackman-Harris fourth-term window. The SNR in the frequency domain is the quotient of these spectra.

Recordings of localized impulse responses. Responses to very short $(10 \mu \mathrm{s})$, bright white light flashes (i.e., impulse responses) were recorded from both photoreceptor somata and axons. All of the cells were dark-adapted before the recordings. Because the ISI was only $1 \mathrm{~s}$, the more sensitive and slowly adapting photoreceptors were probably partly light-adapted during these recordings. Because there are spikes or spikelike potentials in the axons (Weckström et al., 1993), these recordings were sampled at $2.5 \mathrm{kHz}$ and not filtered. Electrode locations in the cockroach eye were approximately estimated by using the color differences in the tissue. The part of the retina containing photoreceptor somata and pigment cells is black because of screening pigment granules and photopigments (Butler 1973a,b), the area below the retina containing distal parts of the photoreceptor axons is brown because of pigment granules, and the rest of the axonal area up to the lamina is opalescent or very light brown, depending on the quality of incident light.

Simulations. The hypothesis concerning how a group of variable photoreceptors might work as a population was simulated with Simulink (Mathworks). See the general description in Results and details in the supplemental material (available at www.jneurosci.org). The simulation files are available from the authors on request (e-mail: matti.weckstrom@oulu.fi).

\section{Results}

\section{Variability of light responses}

Light-elicited responses of the cockroach green-sensitive photoreceptors showed (in contrast to photoreceptors in most visual systems) an amazing variability of properties under identical stimulation and adaptation conditions. The studied parameters here include sensitivity (Fig. 2), adaptation speed in terms of membrane voltage decay (Figs. 2, 3), acceptance angle of the receptive field (Fig. 4), and signal-to-noise ratio (Fig. 5). In addition to large structural variability (Butler, 1971, 1973a,b; Ribi, 1977; Ernst and Füller, 1987; Füller et al., 1989), all of these functional properties showed large inter-photoreceptor variation of up to two orders of magnitude. The variability in cockroach photoreceptors was randomly distributed all over the eye (the most ventral part and the margin of the eye were not studied). On the basis of previously recorded cells in the same eye, one could never predict which kind of cell would be next penetrated by the electrode. Within a distance of few tens of micrometers in the retina, all extremes of functional variability could be encountered. Also, all of the variability was independent of the diurnal cycle or sex.

\section{Variability of sensitivity and adaptation speed}

Recordings of the photoreceptor sensitivity and adaptation speed were obtained from 200 different dark-adapted photoreceptor cells from 40 cockroaches. There was large variation in both the sensitivity of the photoreceptors (Fig. 2) and the timescale of the voltage decay of the light response (i.e., adaptation speed) (Figs. 2,3 ). The responses to $300 \mathrm{~ms}$ light pulses varied both in the sensitivity and adaptation speed (Fig. 2). Differences in sensitivity in dark-adapted state could vary even over two orders of magnitude (Butler and Horridge, 1973b; Weckström et al., 1993). The adaptational diversity of photoreceptors was even clearer in responses to saturating $10 \mathrm{~s}$ light pulses (Fig. 3). After the initial faster voltage decay the "nonadapting" photoreceptors reached a steady-state depolarization close to the maximum response (Figs. 2 , top row; $3 A, B$, top red trace), whereas the "adapting" cells reached a steady-state depolarization somewhere in the middle of the maximum response and the resting potential (Figs. 2, middle row; $3 A, B$, black traces). Note also that the timescale of the voltage decay inside the adapting cells varied greatly; some reached a depolarization steady state very quickly (in less than a second), and for some not even $10 \mathrm{~s}$ was enough. In addition there were "hyperadapting" photoreceptors, which returned to a state reminiscent of dark adaptation. They repolarized close to the resting potential (Figs. 2, bottom row; $3 A$, bottom red trace) even in bright light and showed discrete depolarizing responses on the tail of the voltage decay (Fig. $3 A$, inset). We cannot identify the mechanism producing these discrete events. However, their shape is quantum bump-like, and therefore, they could be the 

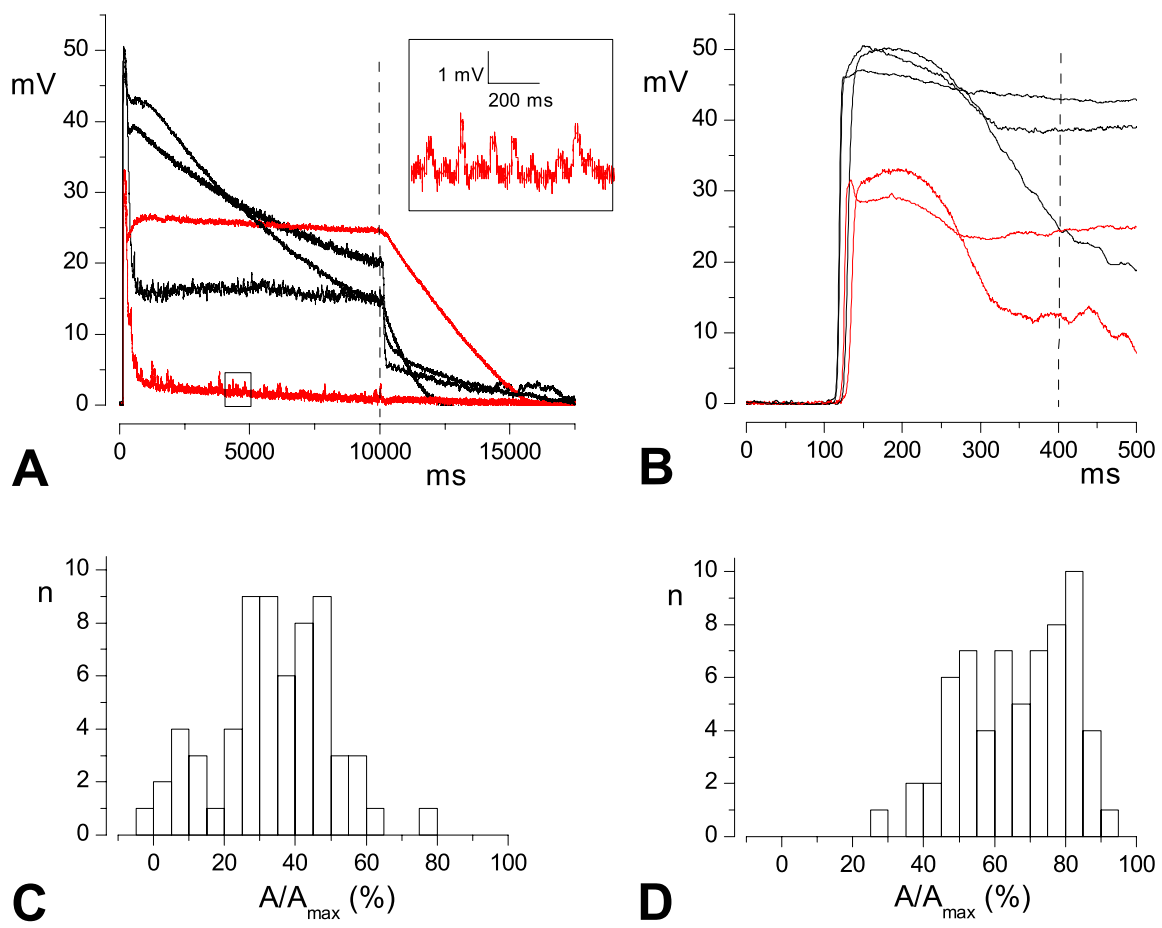

Figure 3. Variability in long light responses. A, Voltage responses to saturating 10 s light pulses from different photoreceptors. There are obviously at least two different phases in the adaptation process (fast and slow), and their relative amounts vary from cell to cell. After the fast phase of adaptation, the cells we call hyperadapting return to a state resembling dark adaptation and show distinguishable discrete events (possibly quantum bumps; see inset), although the bright stimulus light is still on. Stimulus onset was at $100 \mathrm{~ms}$. The red traces highlight the nonadapting (top red trace) and hyperadapting (bottom red trace) responses (for additional explanation, see Results). Note also the differences in light-off responses. $\boldsymbol{B}$, The first $500 \mathrm{~ms}$ (fast phase of adaptation) of the responses in $\boldsymbol{A}$. Stimulus onset and the red traces as in $\boldsymbol{A}$. C, Distribution (total number of cells, $n=64$ ) of the residual relative response amplitude ( $A / A_{\max }$; each response is scaled to its own maximum) at $9.9 \mathrm{~s}$ after stimulus onset $(\boldsymbol{A}$, dashed vertical line). $\boldsymbol{D}$, Distribution of the residual relative response amplitude at $300 \mathrm{~ms}$ after stimulus onset ( $\boldsymbol{B}$, dashed vertical line).
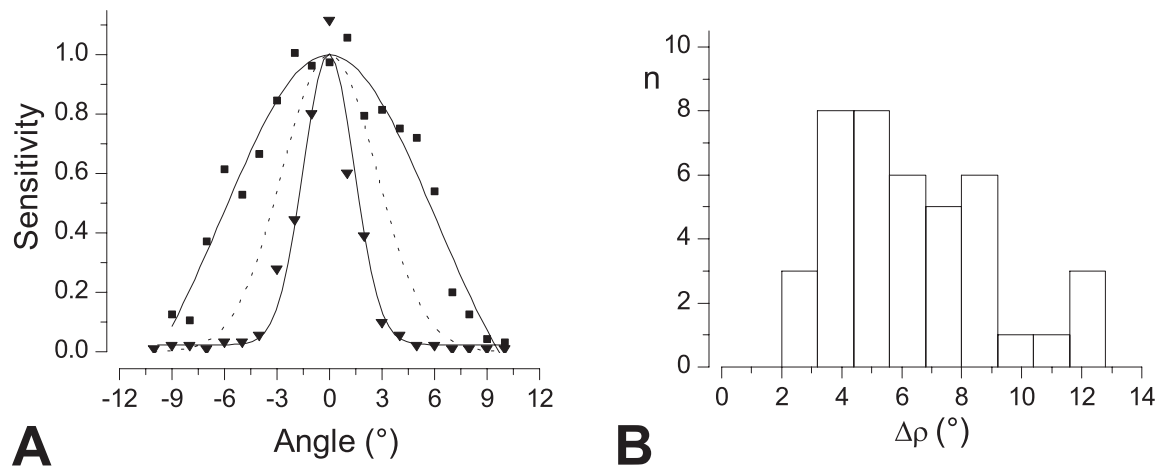

Figure 4. Variability in angular sensitivity. $\boldsymbol{A}$, Recorded data points and fitted curves show large variability in the size of the receptive fields of dark-adapted photoreceptors. On average, the acceptance angle $(\Delta \rho)$ is $6.4^{\circ}\left(\mathrm{SD}, 2.7^{\circ} ; n=41\right)$. The corresponding average Gaussian is shown as a dashed curve. The measured extreme receptive fields at their widest (squares; $\Delta \rho=$ $12.7^{\circ}$ ) and at their narrowest (triangles; $\Delta \rho=2.2^{\circ}$ ) are shown with their Gaussian fits (solid curves). To aid the comparison, the angular sensitivities are scaled to obtain the same maximum sensitivity for all of the Gaussians. This necessarily results in an angular sensitivity $>1$ for some of the data points. $B$, Distribution of $\Delta \rho$ (from recorded data with linear fill; bin width, $1.2^{\circ}$ ) for all of the recorded cells $(n=41)$.

results of a very efficient gain control, in which the number of transduced photons is very small despite the continuing bright light stimulation. In addition, the relaxation speeds in the lightoff responses varied. Although we have divided the photoreceptors in the before-mentioned groups according to their functional properties, these in fact form a continuum (Fig. 3C,D), and we are making this kind of categorization here for demonstration purposes only.
To examine the variability of the light response voltage decay (i.e., adaptation speed) in more detail, we chose two time points in the $10 \mathrm{~s}$ light responses for a closer inspection and calculated the amount of the residual response amplitude relative to maximum amplitude $\left(A / A_{\max }\right.$; percentage) at $9.9 \mathrm{~s}$ and $300 \mathrm{~ms}$ after stimulus onset (Fig. $3 A, B$, dashed vertical lines). Distribution of $A / A_{\max }$ is shown for $9.9 \mathrm{~s}$ in Figure $3 C(n=64$; bin width, $5 \%$; mean \pm SD, $34 \pm 16 \%$ ) and for $300 \mathrm{~ms}$ in Figure $3 D$ (as in Fig. $3 C$; but mean $\pm S D$, $65 \pm 15 \%$ ). The negative bin in Figure 3C is attributable to a hyperadapting cell that hyperpolarized below the resting potential during stimulation. Both distributions were normally distributed according to all three tests used (see Materials and Methods). The lowest $p$ value for $9.9 \mathrm{~s}$ was 0.2128 (D'Agostino-Pearson test) and for $300 \mathrm{~ms}$ was 0.0715 (Lilliefors test).

\section{Variability of angular sensitivity}

The horizontal acceptance angle of the receptive field was measured from 41 darkadapted photoreceptors, and its variability between the cells was also found to be large (Fig. 4). To analyze the variability in receptive fields, the distribution of acceptance angles was determined (Fig. 4B). The distribution was normal according to all three tests used (smallest $p=0.1646$ with D'Agostino-Pearson test). No rigorous selection on the basis of the shape of the receptive fields was performed. It can be noted that the widest acceptance angles are likely to result from coupling of neighboring cells attributable to the recording electrode (Smakmann and Stavenga, 1987). These cells seemed to form a separate group (Fig. 4B). However, because there were no means available to control for this phenomenon, all data recorded were analyzed. Collectively, in dark-adapted photoreceptors, the variability was large and no apparent correlation was found between the width of the receptive field and the adaptational type.

\section{Variability of SNR}

SNR in the frequency domain was recorded at two to seven different adapting light intensities from 14 photoreceptors. At least two different types of dynamic behavior were found (Fig. 5). In all nonadapting photoreceptors and in those adapting cells, in which a steady-state depolarization of at least $\sim 10 \mathrm{mV}$ still remained after $2 \mathrm{~min}$ of constant brightlight adaptation, the SNR grew substantially (close to 10-fold at their best) with increasing adaptation intensity and steady-state depolarization (Fig. 5A). However, the SNR of cockroach photoreceptors was always relatively low in comparison with other well studied species [Calliphora (Juusola et al., 1994); Drosophila 

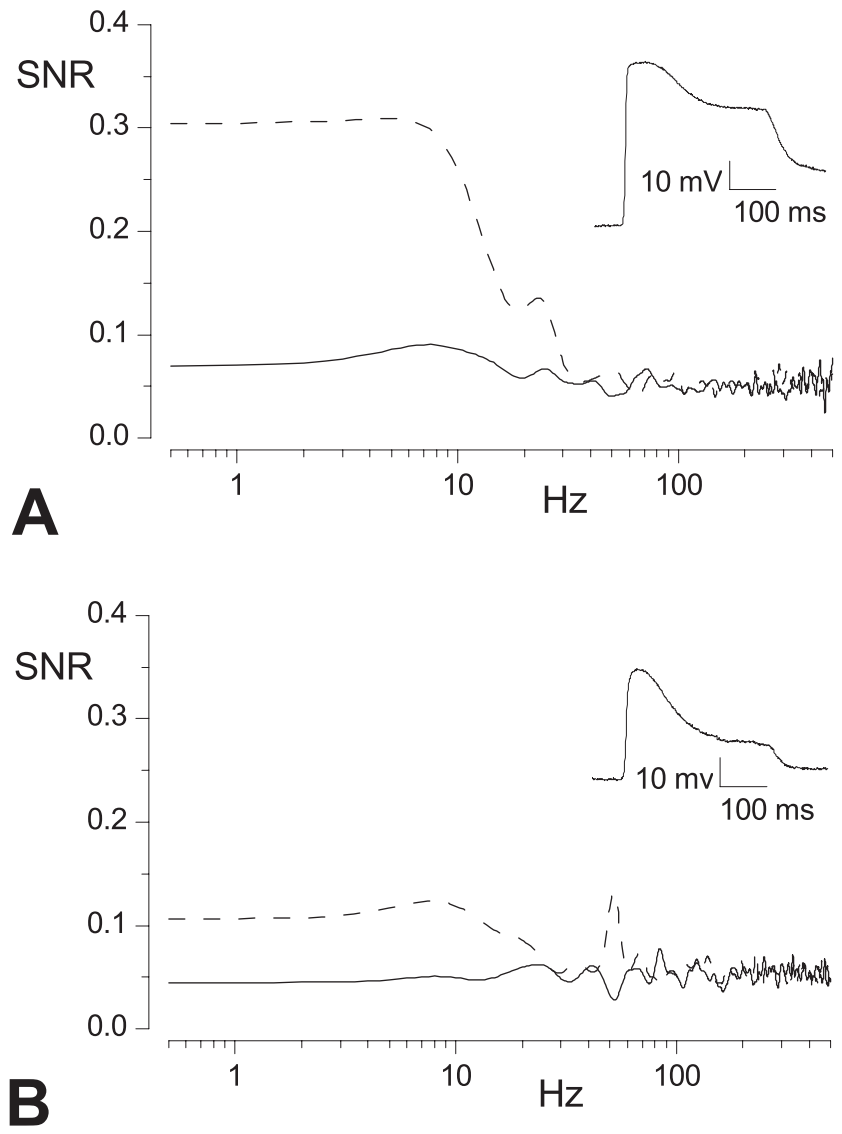

Figure 5. SNR in frequency domain. $A, B$, The SNR is shown for two different photoreceptors at two mean adapting light intensities; a very dim one (solid curves) and a moderately bright one (dotted curves). All plots shown are scaled per unit contrast, although they were measured with a stimulus contrast of 0.16 . Insets show the functional cell types as defined by $300 \mathrm{~ms}$ light responses (compare Fig. 2). $A$, The SNR in all adapting photoreceptors grows substantially with increasing mean stimulus light level and simultaneously increasing steady-state depolarization: the solid curve was measured at a low adapting intensity $(70 \mathrm{ph} / \mathrm{s})$ and the dotted curve at a fairly high intensity $(22,000 \mathrm{ph} / \mathrm{s})$. The nonadapting photoreceptors behave very similarly. $\boldsymbol{B}$, In hyperadapting photoreceptors, the SNR is always low, even in bright illumination (solid curve, $140 \mathrm{ph} / \mathrm{s}$; dotted curve, $14,000 \mathrm{ph} / \mathrm{s}$ ).

(Juusola and Hardie, 2001)]. The SNR was always very low or nearly zero (Fig. $5 B$ ) in all hyperadapting and in those adapting photoreceptors in which the steady-state depolarization reached a value close to resting potential $(<10 \mathrm{mV})$. In the hyperadapting cells, the light response after a 2 min adaptation period consisted of discrete events (possibly quantum bumps) even in bright illumination (compare Fig. $3 A$, bottom trace and inset).

\section{Graded responses in photoreceptor somata and spikes in their axons}

Localized impulse responses from different parts of the photoreceptors were recorded from 23 photoreceptor somata and 11 axons. The axon recordings were technically very demanding, and the axon impalements had a correspondingly short duration. The electrodes need to be the sharper the more proximally one wants to penetrate the axon successfully, and thus we did not succeed with intracellular recordings from the proximal one-half of the axon. The responses were distinct from each other at different locations in the photoreceptors (Fig. 6). In the region of the distal soma, the responses were purely graded $(n=17)$, and in the proximal soma, there were small spikelets in the rising phase (and on the top) of the graded responses $(n=6)$. In the early distal

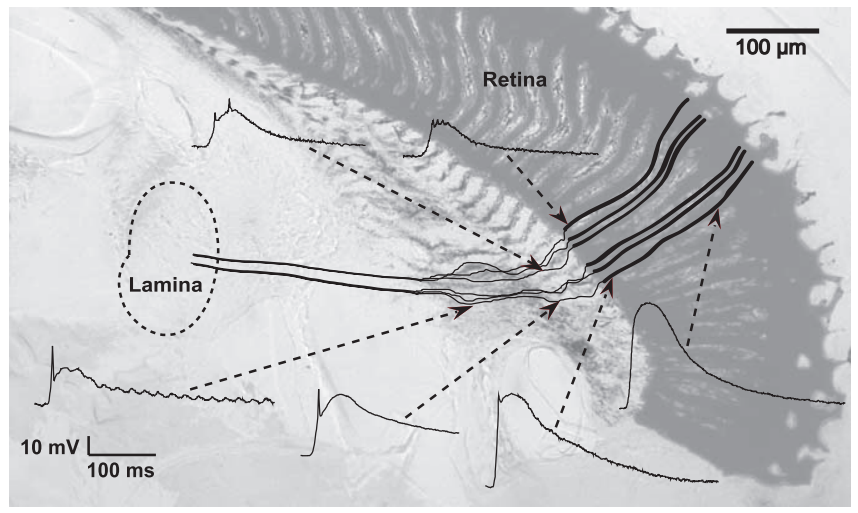

Figure 6. Light responses from different locations in cockroach photoreceptors. Approximate recording locations (marked with arrows) of different impulse responses and the hypothetical pooling of the photoreceptors are drawn on a photographed slice of the cockroach visual tract. The bottom four responses show the main characteristics of the responses and the upper two show the variation in spiking. The photograph was taken through a microscope of an unstained thick slice ( $\sim 50 \mu \mathrm{m}$, fixed with $10 \%$ formaldehyde in buffered insect saline, embedded in Historesin). Recordings were localized by using the color differences in the unstained tissue (see Materials and Methods). Hypothetical drawings of the photoreceptors on top of the photograph are based on our own Lucifer yellow injections (Weckström et al., 1993) and previous structural data of the retina-lamina projections (Ribi, 1977; Ernst and Füller, 1987). Based on the anatomical finding that on their way to the lamina photoreceptor axons regroup and form bundles of $6-20$ axons or more, we hypothesize that these new bundles synapse to the same second-order neuron. The light responses in the distal soma are purely graded, and in the proximal soma there are presumably backpropagated and strongly attenuated action potentials on the rising phase or on top of the graded responses. The responses in the axon are also superimposed by spikes, which are less attenuated. The closer to the soma the recording site is the more attenuated the action potential amplitudes are, and the opposite holds for the graded potentials. The generation site of the action potentials in the axon is unknown.

axons $(n=9)$, the spikes had higher amplitudes, and the graded responses had lower amplitudes than in the soma. The deeper from the axon we recorded $(n=2)$, the bigger was the amplitude of the spike and the smaller the graded response. The graded responses thus attenuated strongly toward the axon terminals, and the spikes attenuated toward the photoreceptor somata (Weckström et al., 1993). There was variation also in spike coding between different photoreceptors, as shown by the variability in the number of spikes (one to four) in the impulse responses (Fig. 6 , topmost responses), but this was not investigated further.

\section{Hypothesis and simulation}

What could be the benefit of the large functional variability of cockroach photoreceptors? The anatomical investigations of the trajectories of photoreceptor axons suggest pooling of several (maybe 6-20) photoreceptor responses on every second-order cell in the first optic neuropil, the lamina (Ribi, 1977; Ernst and Füller, 1987; Füller et al., 1989). The anatomy of the lamina is not known in detail. Unfortunately, intracellular recordings of light responses from the photoreceptor terminals or small postsynaptic neurons in the lamina have been too difficult to be practical with the methods available. Consequently, we approached this problem by means of mathematical simulation. As a starting point for the hypothesis, one has to think about the nature of the light response signals. If all of the photoreceptors carried the same signals, the pooling would undoubtedly reduce noise (Laughlin, 1981, 1990), thereby increasing the SNR. This is the case, for example, in dipteran compound eyes where six photoreceptor axons project into neuro-ommatidium (van Hateren, 1986). However, here we have shown that the cockroach photoreceptor properties vary considerably (Figs. 2-5) and randomly 


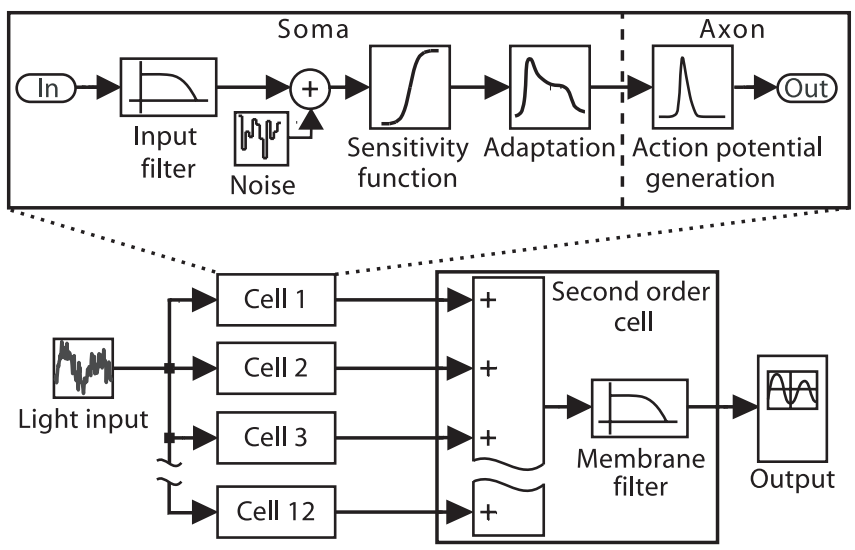

Figure 7. Simulation block diagram. Our program simulates a bundle of 12 different photoreceptor cells, which all pool on the same second-order cell, and produces the hypothetical postsynaptic response as output. The input data sequence (i.e., light stimulus) used in the simulations was 1/fnoise (clipped at zero) produced with AlaNoise (copyright by Alaa Makdissi, Paris, France; www.alamath.com). Differences between the cells are based on our results in this paper, and the number of pooled cells is an average estimate based on previous anatomical findings (Ribi, 1977; Ernst and Füller, 1987; Füller et al., 1989). The block diagram of our simulation shows the parallel and variable photoreceptor cells summing on the same second-order cell. The top panel describes a simulated photoreceptor in more detail.

(Figs. 3C,D , 4B) from cell to cell, and the assumption of identical pooled signals therefore has to be abandoned. Hence, we want to know the performance of a system in which photoreceptors sharing the same postsynaptic cell each carry different information from approximately the same spatial direction (Fig. 6). In addition to pooling of highly variable signals, another essential part of our hypothesis involves spike coding already in the photoreceptor axons (Weckström et al., 1993).

Our hypothesis about the function of the cockroach visual system is as follows. Photoreceptors code their light responses into action potentials in their axons (Fig. 6) (Weckström et al., 1993). A group of nearby photoreceptors (maybe 6-20 of them) is pooled into the same second-order neuron. The pooled photoreceptors are randomly different (as described by our results) and produce different spike trains, which thus forms a kind of population coding scheme (Dayan and Abbott, 2001; Averbeck et al., 2006; Jazayeri and Movshon, 2006). The nonadapting or very weakly adapting photoreceptors optimally code light intensities that change slowly or remain constant, and produce most invariable and long-lasting spike trains. The adapting photoreceptors with variable adaptation speeds form a fairly slow, but conventional kind of contrast coding apparatus [compare, for example, to blowfly (Juusola et al., 1994)] and produce spike trains and bursts with largest variation. The hyperadapting cells simply detect transients and produce very sparse spikes. All cells come with variable sensitivities for optimal coding at different average illumination environments.

We tested the pooling hypothesis stated in the previous paragraph by simulating (Simulink; Mathworks) a group of 12 photoreceptors (Fig. 7) (approximately as they were characterized by our recordings) and calculating the resulting second-order cell response. The complexity of the simulation was kept at a minimum, and the cells in it were not intended to represent real models of the actual cells. Each cell in the simulation contained a passive input filter, added independent white noise, a saturating sensitivity function, an adaptation function and a spike generator (Fig. 7, top panel). The adaptation function was merely a mathematical operation, which caused membrane responses to repo- larize under constant illumination, mimicking recorded photoreceptor properties. The spike generator was a pulse generator equipped with a threshold, a refractory period, and a low-pass filter to give the pulses a more realistic shape. In the simulations, the inter-photoreceptor variability was achieved by varying the gain of the sensitivity function, and the time constant and extent of repolarization (i.e., adaptation). The first synapse was simulated as a linear summation of the signals of the 12 parallel photoreceptors and a passive membrane (postsynaptic) filter. However, in the real system, both membranes are likely to be actively filtering the signals. Likewise, the adaptation processes form a much more elaborate complex in modulating the responses. Therefore, the real second-order neurons are likely to perform better because of matched filtering of the signals (cf. Laughlin, 1996). The properties of the pooled photoreceptors were varied randomly in 50 simulation runs of $11 \mathrm{~s}$ each. The more the properties were varied, the more reliably the response of the secondorder cell followed the stimulus. In our simulations, the variation in these properties mimicked the observed variability. The simulated responses of a single postsynaptic cell were pooled from responses of nonidentical (i.e., variable) photoreceptors, and compared with those of pooled identical cells (Fig. 8). The SNR is not a good measure for this comparison, because the noise level in photoreceptors is arbitrary and the signals are coded into action potentials. The performance of pooled photoreceptors was evaluated in terms of the reliability of coding (i.e., how well the output follows the input), calculated as the coefficient of determination $\left(r^{2} ; r\right.$, correlation coefficient) between the filtered input and the output. The reliability in the case of the pooled nonidentical cells was $0.83 \pm 0.05$ (mean \pm SD). This was higher than with any type of pooled identical cells, which in the best case was 0.67. Especially, when the range of input amplitudes is large (i.e., contrasts are high), the system of pooled variable cells performs quite well both in dim and fairly brightly illuminated environment (Fig. 8C). However, pooled identical cells do much worse, especially in dim conditions. Simulation runs without spike generation showed no significant difference between the reliability of pooled identical and nonidentical cells.

\section{Discussion}

Neurons in insect nervous systems are often assumed to be precisely responding and structurally rigid. Several investigations report small inter-photoreceptor variability of functional parameters. In blowflies, the variability of R1-6 photoreceptor sensitivity, measured as SD of the position of the $V$-log- $I$ function, is $50 \%$ or less (Hardie, 1979). Variability of frequency response is $\sim 20 \%$ as SD of the $-3 \mathrm{~dB}$ high-frequency cutoff (Juusola et al., 1994). The acceptance angle of photoreceptors varies $\sim 20 \%$ in flies and $\sim 25 \%$ in dragonflies (Laughlin and Hardie, 1978). A work on 46 butterfly species (Eguchi and Horikoshi, 1984) reports a 10-20\% variability of sensitivity, as SD of the parameters of Naka-Rushton equation. Compound eyes of these insect groups are structurally rigid and ommatidia with hexagonal lenses form regular rows and columns. The situation in the cockroach is different. Previous studies indicate a large degree of variation in structure of the compound eye and photoreceptors (Butler, 1971, 1973a,b; Ribi, 1977; Ernst and Füller, 1987; Füller et al., 1989; Ferrell and Reitcheck, 1993). Lenses and rhabdoms have variable sizes and shapes. Photoreceptors vary in size, shape and location and size of their rhabdomeres. Photoreceptor axons are long and project in an irregular manner toward their synaptic terminals. Until now, there has been no reasonable explanation for this apparently random structural variation. 
Sensitivity variation has been reported in cockroach photoreceptors previously (Butler, 1973b). Furthermore, green- and UV-sensitive photoreceptors (Mote and Goldsmith, 1970, 1971) show different light response dynamics (Mote, 1990). Here, we showed that the green-sensitive photoreceptors exhibit large variability in several functional aspects: sensitivity and adaptation (Figs. 2, 3), receptive field size (Fig. 4), and SNR (Fig. 5). All tested properties (Figs. 3, 4) were normally distributed, suggesting random variability. The findings are surprising, given the results from other insects (Laughlin, 1981), like flies (Juusola et al., 1994) and even nocturnal bees (Warrant et al., 2004). In those species, the compound eye is ordered, and functional cell-to-cell variation is very small, although regional specializations occur (Burton et al., 2001). We demonstrated that cockroach photoreceptors use spike coding in axons (Fig. 6) (Weckström et al., 1993) and suggest a functional justification for the large variability and spike coding in form of a computer simulation (Figs. 7, 8), which should be considered a hypothesis rather than a realistic model of the system.

\section{How is variability achieved?}

Detailed anatomical studies (Butler, 1971, 1973b; Ferrell and Reitcheck, 1993) show large variation in the cockroach optical system. Size and extent of the rhabdom vary, suggesting variation in photon capture efficiency, leading to variability in photoreceptor sensitivity, which is indeed found experimentally (Fig. 2) (Butler and Horridge, 1973b; Weckström et al., 1993).

In the more or less diffraction-limited compound eye, the ratio of axial distance between the lens and the rhabdom to the width of the rhabdom tip determines angular sensitivity (Snyder, 1977; Stavenga, 2003). The anatomical data (see above) point to large variation in those parameters as well. Additional variability in the optical structure exists inside the photoreceptors. Palisade formation and screening pigment migration (Butler, 1971, 1973b), regulating both overall and angular sensitivity of photoreceptors (Snyder and Horridge, 1972), show a circadian rhythm but also variation at any phase of the rhythm (Ferrell and Reitcheck, 1993).

The inter-photoreceptor variability of adaptation speed (Figs. 2,3 ) is difficult to explain. One factor could be sensitivity variation, because sensitivity is coupled to adaptation processes. However, adaptational variability seems to exist independently of sensitivity differences (Fig. 2). Palisade formation and pigment migration participate in adaptational changes of sensitivity, but there is no information about the speed of these processes in the cockroach. Their variability (Ferrell and Reitcheck, 1993) might explain part of the variation in the speed of the slow phase of adaptation (Fig. 3A). Other possible explanations include the phototransduction (for review, see Hardie and Raghu, 2001) (e.g., variability in calcium sensitivity of key enzymes, differences in potassium channel types and distribution) (Laughlin and Weckström 1993; Weckström and Laughlin 1995) and variability in membrane resistance and cell size. The time of day when recordings were made (diurnal variation) and the electrode position in the eye (spatial variation) did not have any systematic effects. The initial state of the recorded photoreceptor, influenced by stimulation protocols (like the ISI used), is more problematic. Most sensitive photoreceptors need several minutes for proper dark adaptation even after one flash. However, the same interphotoreceptor variability remained whatever ISI was used, although adaptation speed was influenced by preceding illumination.

\section{Variability and seeing at low luminance}

The structural variation, the large variability of light responses (Figs. 2-5), and the apparent functional randomness (Figs. 3C,D, $4 B$ ) may prevent formation of a coherent picture of the animal's surroundings. However, cockroaches use vision for behavioral guidance (Riemay, 1984; Kelly and Mote, 1990) and antennal steering (Ye et al., 2003). At low illumination, the random variation of photoreceptor properties might be beneficial, especially, when light-elicited voltage responses are coded into action potentials already in the axons (Fig. 6) (Weckström et al., 1993). The variability and spike coding of light responses, a seemingly 
unique combination among insects (cf. Warrant et al., 2004; Greiner, 2006), form a fairly efficient visual system at low illumination conditions by using population coding (Fig. 8).

The population coding hypothesis is supported by anatomical data. There are $\sim 26,000-45,000$ photoreceptors in the cockroach compound eye (Füller et al., 1989). The small size of lamina (Ribi, 1977; Ernst and Füller, 1987) and the number of putative third-order neurons $(\sim 3000)$ (Füller et al., 1989) gives only 2000-3000 second-order neurons per compound eye. Thus, there is a great amount of pooling in the first synapse, $\sim 6-20$ to one. Some photoreceptors synapse directly in the second neuropil, the medulla (Ribi, 1977; Ernst and Füller, 1987), but the same considerations hold there as well. The connectivity of photoreceptor axons is irregular, with axon groups regrouping in a seemingly random manner between retina and the first neuropil (Fig. 6). This means random pooling of signals and degrading spatial resolution (French et al., 1977).

The SNR in the cockroach photoreceptor soma (graded response) is small at low illumination (Fig. 5), as in other species (Laughlin, 1990; Juusola et al., 1994; Juusola and Hardie, 2001). Our simulations suggest that action potential coding in photoreceptors with varying properties can perform better than any single photoreceptor type alone (Fig. 8). When similar photoreceptors carrying nearly identical information are pooled on the same second-order neuron, the reliability is poor. However, reliability is improved when functionally different receptors carrying different information from approximately the same spatial direction are pooled on the same second-order cell. Pooling naturally reduces spatial resolution (Laughlin, 1981, 1990; Warrant et al., 2004).

Higher reliability of signaling of pooled different, as opposed to pooled identical, photoreceptor cells can be explained by larger voltage operating range (avoiding saturation) and more faithful coding of the stimulus. A rich stimulus is matched by nearly equal richness of the photoreceptor properties. The presence of action potentials creates a stochastic resonance-like phenomenon (Collins et al., 1995; Wiesenfeld and Moss, 1995), whereby the system works more sensitively with noise than without. The poor SNR of individual photoreceptors (Fig. 5) is bypassed by coding transient events into spikes. Although the SNR in frequency domain works well with flies (Juusola et al., 1994; Juusola and Hardie, 2001), it is mostly unsuitable for describing cockroach photoreceptor function, because, after proper light adaptation, many cells produce very small signals or only quantum bump-like events (compare Fig. 3A), leading to a small SNR.

The inter-photoreceptor variability and the proposed pooling strategy differ essentially from the kind of variability produced by noise. Large noise degrades signaling. By pooling, this can be reduced, but only in proportion to the square root of the number of cells pooled. In the present scheme, this SNR improvement is seen at low illumination in which some cells necessarily respond to single photons. Noise as such is not able to increase the operational voltage or sensitivity range of the cells as does variability. The variability in sensitivity, light-to-voltage gain, and adaptation speed makes the dynamic coding of wide range of light intensities possible, assuming that it is important for the animal to distinguish changes in light signals both in bright and dim environments. This is a simplification, and the real transformations taking place in the photoreceptor-to-second-order cell transmission have to wait for additional investigations. The advantage of the cockroach system of pooling over, for example, pooling in neural superposition eye of Dipteran flies (Kirschfeld, 1967) is clearly the increase of functional operating range, whereas in flies the pooling only increases SNR (Howard et al., 1987). Pooling of photoreceptors in vertebrate retina (Baylor et al., 1971; Wu, 1994) increases SNR and gain of synaptic transmission, with reduction of spatial resolution. With spike and population coding, the pooling of photoreceptor signals in the cockroach is clearly different.

\section{References}

Averbeck BB, Latham PE, Pouget A (2006) Neural correlations, population coding and computation. Nat Rev Neurosci 7:358-366.

Baylor DA, Fuortes MG, O'Bryan PM (1971) Receptive fields of cones in the retina of the turtle. J Physiol (Lond) 214:265-294.

Burton BG, Tatler BW, Laughlin SB (2001) Variations in photoreceptor response dynamics across the fly retina. J Neurophysiol 86:950-960.

Butler R (1971) The identification and mapping of spectral cell types in the retina of Periplaneta americana. Z Vergl Physiol 72:67-80.

Butler R (1973a) The anatomy of the compound eye of Periplaneta americana L. 1. General features. J Comp Physiol 83:223-238.

Butler R (1973b) The anatomy of the compound eye of Periplaneta americana L. 2. Fine structure. J Comp Physiol 83:239-262.

Butler R, Horridge GA (1973a) The electrophysiology of the retina of Periplaneta americana L. 1. Changes in receptor acuity upon light/dark adaptation. J Comp Physiol 83:263-278.

Butler R, Horridge GA (1973b) The electrophysiology of the retina of Periplaneta americana L. 2. Receptor sensitivity and polarized light sensitivity. J Comp Physiol 83:279-288.

Collins JJ, Chow CC, Imhoff TT (1995) Stochastic resonance without tuning. Nature 376:236-238.

Dayan P, Abbott LF (2001) Theoretical neuroscience. Cambridge, MA: MIT.

Eguchi E, Horikoshi T (1984) Comparison of stimulus-response (V-logI) functions in five types of lepidopteran compound eyes (46 species). J Comp Physiol A Neuroethol Sens Neural Behav Physiol 154:3-12.

Ernst A, Füller H (1987) Zur Feinstruktur der Lamina Ganglionaris von Periplaneta americana (L.). Zool Jahrb Anat 115:393-416.

Exner S (1891) Die Physiologie der facettiirten Augen von Krebsen und Insecten. Reprint (Hardie R, translator). The physiology of the compound eyes of insects and crustaceans. Berlin: Springer, 1989.

Ferrell BR, Reitcheck BG (1993) Circadian changes in cockroach ommatidial structure. J Comp Physiol A Neuroethol Sens Neural Behav Physiol 173:549-555.

French AS, Snyder AW, Stavenga DG (1977) Image degradation by an irregular retinal mosaic. Biol Cybern 27:229-233.

Füller H, Eckert M, Blechschmidt K (1989) Distribution of GABA-like immunoreactive neurons in the optic lobes of Periplaneta americana. Cell Tissue Res 255:225-233.

Greiner B (2006) Adaptations for nocturnal vision in insect apposition eyes. Int Rev Cytol 250:1-46.

Hardie RC (1979) Electrophysiological analysis of fly retina. I: Comparative properties of R1-6 and R7 and R8. J Comp Physiol A Neuroethol Sens Neural Behav Physiol 129:19-33.

Hardie RC, Raghu P (2001) Visual transduction in Drosophila. Nature 413:186-193.

Howard J, Blakeslee B, Laughlin SB (1987) The intracellular pupil mechanism and photoreceptor signal: noise ratios in the fly Lucilia cuprina. Proc R Soc Lond B Biol Sci 231:415-435.

Jazayeri M, Movshon JA (2006) Optimal presentation of sensory information by neural populations. Nat Neurosci 9:690-696.

Juusola M, Hardie RC (2001) Light adaptation in Drosophila photoreceptors. I. Response dynamics and signalling efficiency at $25^{\circ} \mathrm{C}$. J Gen Physiol 117:3-25.

Juusola M, Kouvalainen E, Järvilehto M, Weckström M (1994) Contrast gain, signal-to-noise ratio, and linearity in light-adapted blowfly photoreceptors. J Gen Physiol 104:593-621.

Juusola M, Uusitalo R, Weckström M (1995) Transfer of graded potentials at the photoreceptor interneuron synapse. J Gen Physiol 105:117-148.

Kelly K, Mote MI (1990) Avoidance of monochromatic light by the cockroach Periplaneta americana. J Insect Physiol 36:287-291.

Kirschfeld K (1967) Die Projektion der optischen Umwelt auf das Raster der Rhabdome im Komplexauge von Musca. Exp Brain Res 3:248-270.

Kouvalainen E, Weckström M, Juusola M (1994) A method for determining 
photoreceptor signal-to-noise ratio in the time and frequency domains with a pseudorandom stimulus. Vis Neurosci 6:1221-1225.

Laughlin SB (1981) Neural principles in the peripheral visual systems of invertebrates. In: Handbook of sensory physiology, Vol. VII/6B (Autrum H, ed), pp 133-280. New York: Springer.

Laughlin SB (1990) Invertebrate vision at low luminances. In: Night vision (Hess RF, Sharpe LT, Nordby K, eds), pp 223-250. Cambridge, UK: Cambridge UP.

Laughlin SB (1996) Matched filtering by a photoreceptor membrane. Vision Res 36:1529-1541.

Laughlin SB, Hardie RC (1978) Common strategies for light adaptation in the peripheral visual systems of fly and dragonfly. J Comp Physiol A Neuroethol Sens Neural Behav Physiol 128:319-340.

Laughlin SB, Weckström M (1993) Fast and slow photoreceptors-a comparative study of the functional diversity of coding and conductances in the Diptera. J Comp Physiol A Neuroethol Sens Neural Behav Physiol 172:593-609.

Mote MI (1990) Structure and function of the visual systems of the Am cockroach. In: Cockroaches as models for neurobiology: applications in biomedical research, Vol II (Huber I, Masler EP, Rao BR, eds), pp 203 224. Boca Raton, FL: CRC.

Mote MI, Goldsmith TH (1970) Spectral sensitivities of color receptors in the compound eye of the cockroach Periplaneta. J Exp Zool 173:137-146.

Mote MI, Goldsmith TH (1971) Compound eyes: localization of two color receptors in the same ommatidium. Science 171:1254-1255.

Ribi WA (1977) Fine structure of the first optic ganglion (lamina) of the cockroach, Periplaneta americana. Tissue Cell 9:57-72.

Riemay H (1984) Verhaltensphysiologische Untersuchungen zur Einwirkung des Lichtes auf den Fluchtlauf der Schabe Periplaneta americana (L.). Zool Jb Physiol 88:491-497.

Shaw SR (1990) The photoreceptor axon projection and its evolution in the neural superposition eyes of some primitive brachyceran Diptera. Brain Behav Evol 35:107-125.

Smakmann JGJ, Stavenga DG (1987) Angular sensitivity of blowfly photo- receptors: broadening by artificial electrical coupling. J Comp Physiol A Neuroethol Sens Neural Behav Physiol 160:501-507.

Smola U (1976) Voltage noise in insect visual cells. In: Neural principles in vision (Zettler F, Weiler R, eds), pp 194-213. New York: Springer.

Stavenga DG (1979) Pseudopupils of compound eyes. In: Handbook of sensory physiology, Vol VII/6A (Autrum H, ed), pp 357-439. New York: Springer.

Stavenga DG (2003) Angular and spectral sensitivity of fly photoreceptors. I. Integrated facet lens and rhabdomere optics. J Comp Physiol A Neuroethol Sens Neural Behav Physiol 189:1-17.

Snyder AW (1977) Acuity of compound eyes: physical limitations and design. J Comp Physiol 116:161-182.

Snyder AW, Horridge GA (1972) The optical function of changes in the medium surrounding the cockroach rhabdom. J Comp Physiol 81:1-8.

van Hateren JH (1986) Electrical coupling of neuro-ommatidial photoreceptor cells in the blowfly. J Comp Physiol A Neuroethol Sens Neural Behav Physiol 158:795-811.

Warrant EJ, Kelber A, Gislen A, Greiner B, Ribi W, Wcislo WT (2004) Nocturnal vision and landmark orientation in a tropical halictid bee. Curr Biol 14:1309-1318.

Weckström M, Laughlin SB (1995) Visual ecology and voltage-gated ion channels in insect photoreceptors. Trends Neurosci 18:17-21.

Weckström M, Järvilehto M, Heimonen K (1993) Spike-like potentials in the axons of nonspiking photoreceptors. J Neurophysiol 69:293-296.

Wiesenfeld K, Moss F (1995) Stochastic resonance and the benefits of noise: from ice ages to crayfish and SQUIDs. Nature 373:33-36.

Wu CF, Pak L (1978) Light induced voltage noise in the photoreceptor of Drosophila melanogaster. J Gen Physiol 71:249-268.

Wu SM (1994) Synaptic transmission in the outer retina. Annu Rev Physiol $56: 141-168$.

Ye S, Leung V, Khan A, Baba Y, Comer CM (2003) The antennal system and cockroach evasive behavior. I. Roles for visual and mechanosensory cues in the response. J Comp Physiol A Neuroethol Sens Neural Behav Physiol 189:89-96. 